\title{
The pungent substances piperine, capsaicin, 6-gingerol and polygodial inhibit the human two-pore domain potassium channels TASK-1, TASK-3 and TRESK
}

\author{
Leopoldo R. Beltrán ${ }^{1}$ *, Corinna Dawid ${ }^{2}$, Madeline Beltrán ${ }^{1}$, Guenter Gisselmann ${ }^{1}$, \\ Katharina Degenhardt ${ }^{2}$, Klaus Mathie ${ }^{2}$, Thomas Hofmann² and Hanns Hatt ${ }^{1}$ \\ ' Department of Cell Physiology, Ruhr-University-Bochum, Bochum, Germany \\ ${ }^{2}$ Chair for Food Chemistry and Molecular Sensory Science, Technische Universität München, Freising, Germany
}

\section{Edited by: \\ Pierangelo Geppetti, University of \\ Florence, Italy}

Reviewed by:

Li Zhang, National Institute on Alcohol Abuse and Alcoholism, National Institutes of Health, USA Robert L. Barkin, Rush University Medical Center/Northshore University Health System, USA

\section{*Correspondence:}

Leopoldo R. Beltrán, Department of Cell Physiology, Ruhr-UniversityBochum, Universitaetsstrabe 150, 44780 Bochum, ND 4-132 Germany e-mail: leopoldo.beltran@rub.de

\begin{abstract}
For a long time, the focus of trigeminal chemoperception has rested almost exclusively on TRP channels. However, two-pore domain $\left(K_{2 P}\right)$ potassium channels have recently been identified as targets for substances associated with typical trigeminal sensations, such as numbing and tingling. In addition, they have been shown to be modulated by several TRP agonists. We investigated whether the pungent substances piperine, capsaicin, 6-gingerol and polygodial have an effect on human $K_{2 P}$ channels. For this purpose, we evaluated the effects of these pungent substances on both wild-type and mutant $K_{2 P}$ channels by means of two-electrode voltage-clamp experiments using Xenopus laevis oocytes. All four pungent substances were found to inhibit the basal activity of TASK-1 $\left(K_{2 P} 3.1\right)$, TASK-3 $\left(K_{2 P}\right.$ 9.1), and TRESK (K $\left.K_{2 P} 18.1\right)$ channels. This inhibitory effect was dose-dependent and, with the exception of polygodial on TASK-1, fully reversible. However, only piperine exhibited an $\mathrm{IC}_{50}$ similar to its reported $\mathrm{EC}_{50}$ on TRP channels. Finally, we observed for TASK-3 that mutating $\mathrm{H} 98$ to $\mathrm{E}$ markedly decreased the inhibition induced by piperine, capsaicin, and 6 -gingerol, but not by polygodial. Our data contribute to the relatively sparse knowledge concerning the pharmacology of $\mathrm{K}_{2 \mathrm{P}}$ channels and also raise the question of whether $\mathrm{K}_{2 \mathrm{P}}$ channels could be involved in the pungency perception of piperine.
\end{abstract}

Keywords: $K_{2 P}$ channels, pungency, piperine, capsaicin, 6-gingerol, polygodial

\section{INTRODUCTION}

In modern cuisine, numerous spices, such as black peppercorns (Piper nigrum), chili pepper (Capsicum annuum), mountain pepper (Tasmannia lanceolata) or ginger (Zingiber officinalis), have been appreciated worldwide for their ability to add a pungent orosensory impression to dishes. Today, after several genetic and functional studies, the pungency of these spices is believed to be based mainly on the activation of two members of the transient receptor potential (TRP) family, TRPV1 and TRPA1. These two polymodic, non-selective cation channels, which also play a role in pain perception, are expressed on free afferent nerve endings of trigeminal neurons in the oral cavity (Calixto et al., 2005).

Over the years, a series of non-volatile phytochemicals, which were able to induce a pungent sensation but varied widely in molecular structure, have been reported to activate these two ion channels. For example, capsaicin, one of the most pungent phytochemicals from chili peppers, was the first agonist reported for TRPV1 (Caterina et al., 1997). Piperine, one of the key molecules responsible for the pungency of black pepper (Dawid etal., 2012), has also been shown to activate TRPV1 (McNamara et al., 2005). Similarly, other pungent Piper nigrum amides such as, e.g., piperolein $\mathrm{B}$, were reported to activate the ion channels TRPA1 and TRPV1 (Correa etal., 2010; Okumura etal., 2010). In the same way, polygodial, the pungent drimane-type sesquiterpene dialdehyde from mountain pepper (Barnes and Loder, 1962), and vanilloids such as, e.g., 6-gingerol from ginger rhizomes (Belitz et al., 2004), have been identified as TRPA1 and TRPV1 activators (Dedov et al., 2002; Witte et al., 2002; Bandell et al., 2004; Calixto et al., 2005; André et al., 2006; Escalera etal., 2008; Iwasaki et al., 2009; Morera et al., 2012).

A vast number of other pungent materials also activate members of the TRP family, e.g., alicine from garlic, eugenol from clove, cinnamaldehyde from cinnamon, and extracts of habanero pepper, Thai green chili or mustard oil (Caterina et al., 1997; Bandell et al., 2004; Calixto et al., 2005). Although the role of TRP channels in chemoperception is well accepted, there are some facts suggesting other possible targets for some of these pungent substances. For example, piperine and capsaicin have been shown to present a stimulus-induced recovery in human psychophysical tests, in which volunteer subjects have reported that, after an initial desensitization period, under recurrent capsaicin or piperine stimulation, the sensation of irritation grew towards un-desensitized levels (Green, 1998). This is in contradiction to TRPV1 responses in heterologous systems (Caterina et al., 1997). Moreover, human beings were found to be able to distinguish between 33 and $330 \mu \mathrm{M}$ of capsaicin, although these concentrations are much higher than those needed for TRPV1 saturation (Green, 1996). 
Two-pore domain $\left(\mathrm{K}_{2} \mathrm{P}, \mathrm{KCNK}\right)$ channels are plausible candidates for a complementary role in the chemoperception of these pungent substances. They are potassium-selective channels that tend to be constitutively open and are thought to be responsible for the leak potassium current present in every excitable cell. $\mathrm{K}_{2 \mathrm{P}}$ channels allow a constant efflux of $\mathrm{K}^{+}$, which helps to maintain the cell at hyperpolarized potentials and may even counterbalance excitatory stimuli (Goldstein et al., 2001; Enyedi and Czirják, 2010). Recently, in rat trigeminal neurons, three members of this family, namely rTREK-1 ( $\mathrm{K}_{2 \mathrm{P}} 2.1$, KNCK2), rTREK-2 $\left(\mathrm{K}_{2 \mathrm{P}} 10\right.$, KCNK10) and rTRAAK ( $\left.\mathrm{K}_{2 \mathrm{P}} 4, \mathrm{KCNK} 4\right)$, have been shown to coexpress with TRP channels in the same neurons (Yamamoto et al., 2009). In mice, mTREK-1 ( $\left.\mathrm{K}_{2 \mathrm{P}} 2, \mathrm{KCNK} 2\right)$ and mTRAAK $\left(\mathrm{K}_{2 \mathrm{P}} 4\right.$, KCNK4) have been reported to cooperate with TRP channels for the appropriate perception of ambient temperature, meaning that mice lacking both TREK-1 and TRAAK exhibited heat and cold hyperalgesia (Noël et al., 2009). Furthermore, we have recently shown that human TREK-1, TRAAK, and TREK-2 are activated by $2-\mathrm{APB}$, a blocker but also a common activator for several TRP channels (Beltrán et al., 2013), adding this compound to the list of TRP agonists/antagonists that have an effect on $\mathrm{K}_{2 \mathrm{P}}$ channels, e.g., $\mathrm{pH}$, temperature, anandaminde, etc. (Enyedi and Czirják, 2010). In addition to these facts, three $\mathrm{K}_{2 \mathrm{P}}$ channels, namely mTASK-1 $\left(\mathrm{K}_{2 \mathrm{P}} 3, \mathrm{KCNK} 3\right)$, mTASK-3 $\left(\mathrm{K}_{2 \mathrm{P}} 9, \mathrm{KCNK} 9\right)$ and mTRESK $\left(\mathrm{K}_{2 \mathrm{P}} 18\right.$, KCNK 18), have been identified in mice as the molecular targets of hydroxy- $\alpha$-sanshool, a substance that induces typical trigeminal sensations, such as numbing and tingling (Bautista et al., 2008). Despite all these findings, there have been almost no further studies on the role that KCNK channels may play as receptors for other substances affecting the trigeminal system and, although they are now regarded as key players in processes ranging from apoptosis and oxygen chemoperception to depression (Enyedi and Czirják, 2010), little is known about their pharmacology. Therefore, we studied the effects of four dietary pungent substances, namely capsaicin, 6-gingerol, piperine and polygodial (Figure 1), on the human orthologues of the six above mentioned $\mathrm{K}_{2 \mathrm{P}}$ channels and found that piperine inhibited human TASK-1, TASK-3 and TRESK in a dose-dependent manner, exhibiting an $\mathrm{IC}_{50}$ for TASK-1 similar to its reported $\mathrm{EC}_{50}$ on TRPV1. The other 3 substances were also able to induce a dose-dependent inhibition on TASK-1, TASK-3 and TRESK, but only at concentrations relatively

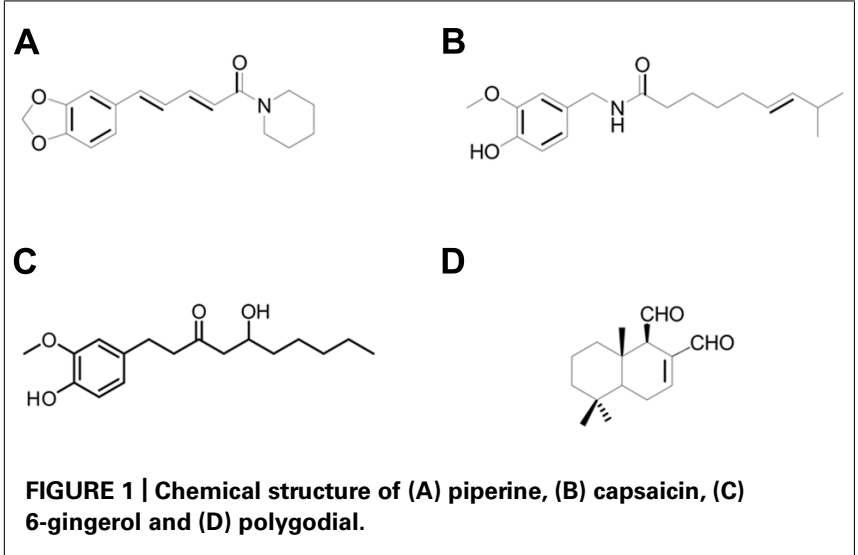

higher than their reported $\mathrm{EC}_{50} \mathrm{~s}$ on TRP channels. Interestingly, we also observed that for TASK-3, mutating H98 to E markedly decreases the inhibition induced by piperine, capsaicin and 6-gingerol.

\section{METHODS \\ MOLECULAR BIOLOGY}

Human TASK-1 ( $\mathrm{K}_{2 \mathrm{P}} 3.1$, KCNK3) cloned into the expression vector pIRES-CD8 was a gift from Dr. Fabrice Duprat (Institut National de la Santé et de la Recherche Médicale, Antipolis, France), and hTRESK (K $2 \mathrm{P} 18.1, \mathrm{KCNK} 18)$, cloned into the plasmid pEXO, was a gift from Prof. Dr. P. Enyedi (Semmelweis University, Budapest, Hungary). These plasmids were linearized with the restriction enzymes BamHI and $\mathrm{XbaI}$, respectively, and then used as templates for in vitro transcription. hTASK-3 (K $2 \mathrm{P} 9.1, \mathrm{KCNK} 9)$ cloned into the expression vector pCR4-TOPO, hTRAAK $\left(\mathrm{K}_{2 \mathrm{P}} 4.1, \mathrm{KCNK} 4\right)$ cloned into pBluescript SK+, hTREK-2 ( $\mathrm{K}_{2 \mathrm{P}} 10.3$, a slightly longer isoform of KCNK10 with a distinct $N$-terminus compared to isoform 1 ; Staudacher et al., 2011) cloned into pCR4-TOPO and hTREK-1 $\left(\mathrm{K}_{2 \mathrm{P}} 2\right.$, KCNK2) cloned into pDNR-dual were purchased from Imagenes (Berlin, Germany). hTREK-1 and hTREK-2 cDNA inserts were later subcloned into pSGEM. The plasmids were linearized with the restriction enzymes SspI for pCR4-TOPO, NotI for pBluescript SK+ and PacI for hTREK-1 and hTREK-2 subcloned into $\mathrm{pSGEM}$, to generate a linear cDNA template for in vitro transcription.

Capped RNAs were synthesized in the presence of the capping analog $\mathrm{m} 7 \mathrm{G}\left(5^{\prime}\right) \mathrm{ppp}\left(5^{\prime}\right) \mathrm{G}$ using the AmpliCap-T7 Message Maker kit (Epicentre, Madison, USA). cRNA was dissolved in nucleasefree water to give a final concentration of $100 \mathrm{ng} / \mu \mathrm{L}$. Mutants or chimeras for hTASK-3 were constructed by standard overlap extension PCR methods and cloned into the pCDNA3 vector.

\section{Xenopus laevis 00CYTES PREPARATION}

The expression in Xenopus laevis oocytes was performed essentially as described by Vogt-Eisele et al. (2007). Briefly, mature female Xenopus laevis frogs were anesthetized with MS-222 at 0.15\%, and surgery was performed according to standard methods. The extracted ovarian lobes were placed into $\mathrm{Ca}^{2+}$-free Barth's solution containing collagenase type II (Worthington Biochemical Corporation, Lakewood, NJ, USA) and kept for $90 \mathrm{~min}$ on a shaker at $40 \mathrm{rpm}$ (room temperature). After that time, healthy stage $\mathrm{V}$ and VI oocytes were selected for injection with cRNA coding for the proteins of interest. For each channel, $30 \mathrm{~nL}$ of cRNA (100 ng/ $\mu \mathrm{L}$ ) was injected using a Microinjector Micro 4 TM (World Precision Instruments, Sarasota, Florida, USA). After injection, the oocytes were kept in ND96 (96 mM NaCl; $2 \mathrm{mM} \mathrm{KCl;} 1.8 \mathrm{mM} \mathrm{CaCl}$; $1 \mathrm{mM} \mathrm{MgCl}_{2} ; 2.5 \mathrm{mM}$ sodium pyruvate $3 \mathrm{mM}$; HEPES $10 \mathrm{mM}$; $\mathrm{pH} 7.2$ ), and measurements were performed 24 to $72 \mathrm{~h}$ after injection.

The oocytes were placed in a $100 \mu \mathrm{L}$ chamber that was perfused with frog Ringer's solution (115 mM NaCl; $2.5 \mathrm{mM} \mathrm{KCl}$; $1.8 \mathrm{mM} \mathrm{CaCl}_{2}$ and $10 \mathrm{mM}$ HEPES pH 7.2). Chemicals to be evaluated were dissolved in this solution before being manually applied to the oocytes using an automatic pipette (research pro, Eppendorf, Germany). The application time was usually three ramps 


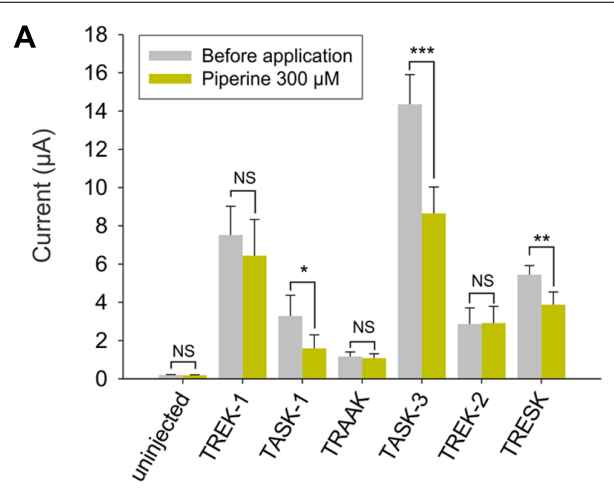

B

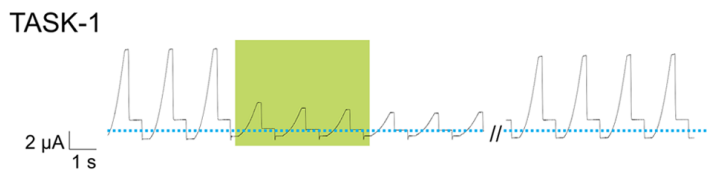

TASK-3

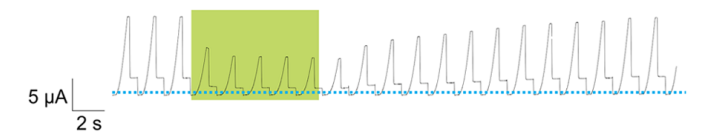

TRESK

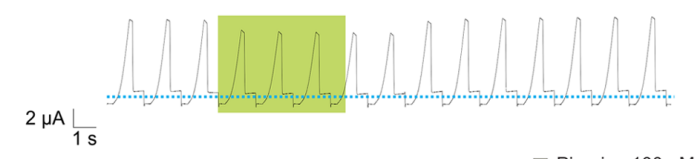

E

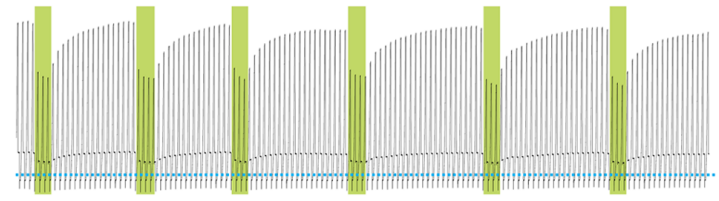

$1 \mu \mathrm{A} \frac{}{20 \mathrm{~s}}$

$\square$ Piperine $30 \mu \mathrm{M}$

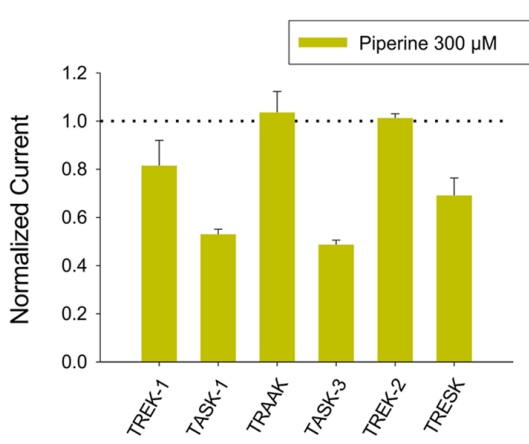

C
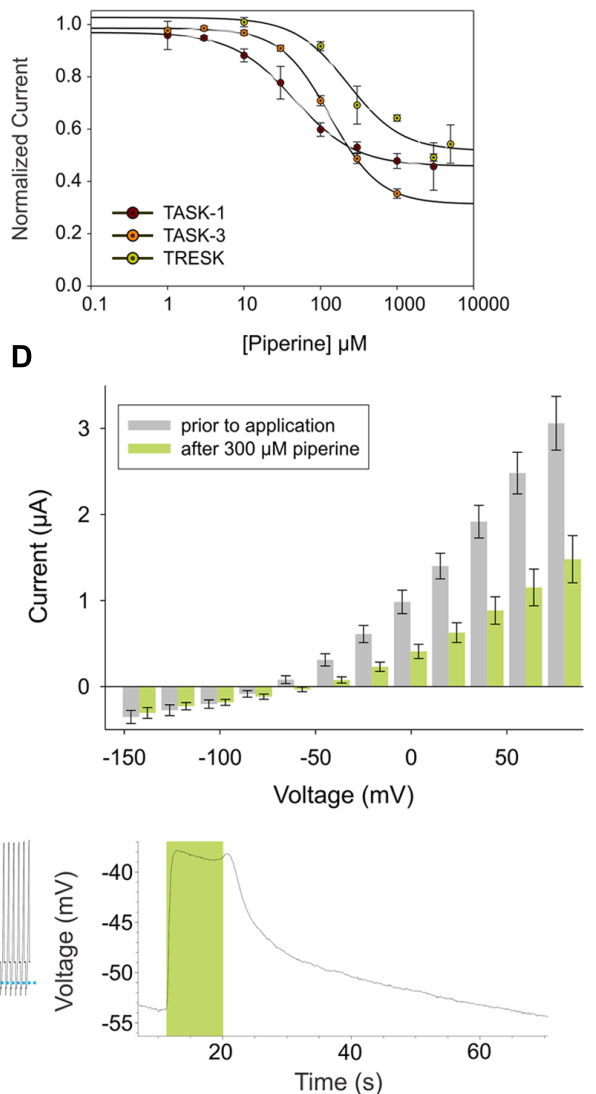

FIGURE 2 | (A) Left (from left to right) absolute currents presented by: uninjected Xenopus oocytes, oocytes injected with cRNA coding for hTREK-1, hTASK-1, hTRAAK, hTASK-3, hTREK-2 and hTRESK, before and after the application of $300 \mu \mathrm{M}$ piperine. The currents were registered at the final $50 \mathrm{~ms}$ of the $+50 \mathrm{mV}$ constant (see methods for a description of the ramp protocol used). Note that the amount of current carried by uninjected oocytes is negligible in comparison to oocytes expressing a $\mathrm{K}_{2 \mathrm{P}}$ channel. Right. Normalized currents showing the effect of $300 \mu \mathrm{M}$ piperine on Xenopus oocytes injected with cRNA coding for hTREK-1, hTASK-1, hTREK-1, hTASK-3, hTREK-2 and hTRESK. For each group, the current was normalized to the current registered prior to the application of $300 \mu \mathrm{M}$ piperine (dotted line). All data are expressed as the mean $+/-$ SEM. $N$ of 3-6 for injected oocytes, $\mathrm{N}$ of 10 for uninjected oocytes. ${ }^{*} P<0.05$, ${ }^{*} P<0.01,{ }^{* *} P<0.001$. (B) Representative voltage clamp recordings of Xenopus laevis oocytes expressing hTASK-1 (top), hTASK-3 (middle) and hTRESK (bottom), before, during and after the application of $100 \mu \mathrm{M}$ piperine. In each case, piperine induced a fully reversible inhibition; for TASK-1, however, due to the prolonged recovery time, 40 ramps were not included. Dotted line represents zero current. (C) Dose-response curves for piperine on hTASK-1 (red circles), hTASK-3 (orange circles) and hTRESK (green circles). The $\mathrm{IC}_{50}$ values are listed in the text and in Table 1. $\mathrm{N}$ of 3-6 oocytes. For each measurement, the current was normalized to the current registered prior to the application of piperine. (D) hTASK-1 currents recorded before (gray bars) and after (green bars) the application of $300 \mu \mathrm{M}$ piperine. The currents were elicited by voltage pulses from -140 to $+80 \mathrm{mV}$ in 20-mV steps, $500 \mathrm{~ms}$ duration from a holding potential of $-80 \mathrm{mV}$. Values were obtained during the last $50 \mathrm{~ms}$ of the voltage pulses. $\mathrm{N}$ of 6 oocytes. (E) Left: representative voltage clamp recording of a Xenopus oocyte expressing hTASK-1 repetitively exposed to $30 \mu \mathrm{M}$ piperine. Right: current clamp recording of a Xenopus oocyte expressing hTASK-1 exposed to $100 \mu \mathrm{M}$ piperine, which led to a membrane depolarization of approximately $15 \mathrm{mV}$. 
Table 1 | IC $C_{50}$ values for pungent substances on human $K_{2 P}$ channels.

\begin{tabular}{llll}
\hline & TASK-1 & TASK-3 & TRESK \\
\hline Piperine & $45 \pm 3.9$ & $133.3 \pm 5.9$ & $230.7 \pm 105$ \\
Capsaicin & $61.1 \pm 4.0$ & $41.8 \pm 3.6$ & $70.2 \pm 5.3$ \\
6-Gingerol & $227.4 \pm 46.5$ & $384.6 \pm 55.6$ & $155.0 \pm 70.9$ \\
Polygodial & $484.5 \pm 18.2$ & $328 \pm 24.7$ & ND \\
\hline
\end{tabular}

All values are expressed in $\mu M$. ND, not determined.

(approximately $10 \mathrm{~s}$ ). For substances that did not show a complete recovery, only one concentration per oocyte was evaluated. For recording, a Turbo Tec-03x (npi electronic GmbH, Tamm, Germany) amplifier was used, with an acquisition rate of $1000 \mathrm{~Hz}$ and a low pass filter of $20 \mathrm{~Hz}$. Borosilicate glass capillaries were pulled with a Kopf vertical pipette puller. The pulled glass capillaries were filled with $3 \mathrm{M} \mathrm{KCl}$.

\section{TWO-ELECTRODE VOLTAGE CLAMP MEASUREMENTS}

A ramp series protocol was used to evaluate the activity of the channels. The voltage ramps consisted of a starting constant of $-100 \mathrm{mV}$ for $300 \mathrm{~ms}$, followed by a ramp to $+50 \mathrm{mV}$ in $700 \mathrm{~ms}$, then a constant at $+50 \mathrm{mV}$ for $150 \mathrm{~ms}$, and a final constant at -40 $\mathrm{mV}$ for $500 \mathrm{~ms}$. The time interval between the ramps was $2 \mathrm{~s}$. To evaluate the effect of an applied substance, we averaged the current registered at the final $50 \mathrm{~ms}$ of the $+50 \mathrm{mV}$ constant in the three ramps showing the maximal response to a substance, and divided that by the average obtained from three ramps prior to the application. In most cases, the currents elicited by this voltage-clamp ramp series protocol were more than ten times larger than the background currents observed in non-injected oocytes, while also presenting the described outward rectification under physiological concentrations of intracellular and extracellular $\mathrm{K}^{+}$(Goldstein et al., 2001; Enyedi and Czirják, 2010). Due to these characteristics, no leakage subtraction was considered necessary. Oocytes not fulfilling these characteristic were not considered for data analysis.

The data were collected using the Cellworks Reader 3.7 software (npi instruments, Tamm, Germany) and analyzed with Clampfit v10.2.0.14 (MDS Analytical Technologies, Sunnyvale, USA). Curve fitting was performed using the 4-parameter Hill equation (Sigmaplot, Systat software inc., San Jose, USA). IC 50 values refer to the concentration at which $50 \%$ of the maximum observed effect was seen. The error associated with these values represents the error in the fit to the mean data at each concentration. Bar diagram data represent the mean $+/-$ SEM. Paired $T$-test was used to evaluate the statistical significance of the results, $P<0.05$ was considered statistically significant and is marked with one star, $P<0.01$ is marked with two stars, and $P<0.001$ is marked with three stars.

\section{DRUGS, CHEMICALS, REAGENTS AND OTHER MATERIALS (INCLUDING SOURCES)}

Capsaicin, DMSO, piperine, and 6-gingerol were purchased from Sigma-Aldrich (Steinheim, Germany), while polygodial was obtained from Santa Cruz Biotechnology (Heidelberg, Germany).
Stock solutions of 100 and $300 \mathrm{mM}$ diluted in DMSO were made and stored at $-20^{\circ} \mathrm{C}$. Aliquots were then taken to obtain the desired concentrations. Piperine and 6-gingerol were purified by means of RP-HPLC in a purity of $>98 \%$.

\section{RESULTS \\ PUNGENT COMPOUNDS INHIBIT TASK-1, TASK-3 AND TRESK IN A DOSE-DEPENDENT MANNER \\ Piperine}

To investigate whether piperine has an effect on human $\mathrm{K}_{2} \mathrm{P}$ channels, we used Xenopus laevis oocytes as expression system for human TREK-1, TASK-1, TRAAK, TASK-3, TREK-2, and TRESK. We then evaluated whether bath-applied piperine could produce an effect on the basal current of these channels. Through this approach, we observed a significant inhibition of TASK-1, TASK-3 and TRESK upon exposure to $300 \mu \mathrm{M}$ piperine (Figure 2A).

All three channels were partially and reversibly inhibited by piperine (Figure 2B). This inhibition was dose-dependent, and, when evaluated at $+50 \mathrm{mV}$, piperine exhibited an $\mathrm{IC}_{50}$ value of $45 \pm 3.9 \mu \mathrm{M}$ on TASK-1, which is in the same range as reported for TRPV1 channels (McNamara et al., 2005). TASK-3 and TRESK showed $\mathrm{IC}_{50}$ values of $133.3 \pm 5.9 \mu \mathrm{M}$ and $230.7 \pm 105 \mu \mathrm{M}$, respectively (Figure $\mathbf{2 C}$ and Table $\mathbf{1}$ ). The current-voltage relationship showed the inhibitory effect of piperine to be more pronounced at potentials more positive than the potassium reversal potential (Figure 2D for TASK-1, data for TASK-3 and TRESK not shown). This is most likely due to the fact that $\mathrm{K}_{2 \mathrm{P}}$ channels behave as outward rectifiers under the given ionic conditions, and the low amount of inward current that they carry is not entirely responsible for the leakage current presented at potentials more negative than the $\mathrm{K}_{\mathrm{rev}}$ (included because no leak substraction was performed). In psychophysical tests, exposure to piperine is reported to lead to desensitization (Green, 1996). This desensitization, however, can be reversed by recurrent stimulation, a phenomenon known as stimulus-induced recovery which does not match the behavior of heterologously expressed TRPV1 when exposed to piperine (McNamara et al., 2005). We therefore assessed the effect of repetitive piperine application on TASK-1, the channel in which it presented its highest potency, but failed to observe any marked difference in the response of TASK-1 to repetitive applications of $30 \mu \mathrm{M}$ piperine (Figure 2E, left). Finally, to study the effect of inhibiting this $\mathrm{K}_{2} \mathrm{P}$ channel at the membrane potential level, Xenopus oocytes expressing TASK-1 were evaluated in currentclamp mode and exposed to piperine, which led to a reversible depolarizing change in the membrane potential (Figure 2E, right).

\section{Capsaicin}

Following the same experimental approach as reported above for piperine, we expressed human TREK-1, TASK-1, TRAAK, TASK-3, TREK-2, and TRESK channels in Xenopus laevis oocytes and evaluated whether bath-application of capsaicin, the pungent principle of chili peppers, could affect the basal current of these channels. TASK-1, TASK-3, and TRESK showed significant decreases in basal activity when exposed to $100 \mu \mathrm{M}$ capsaicin. Interestingly, non-injected oocytes were also significantly inhibited by $100 \mu \mathrm{M}$ 
A

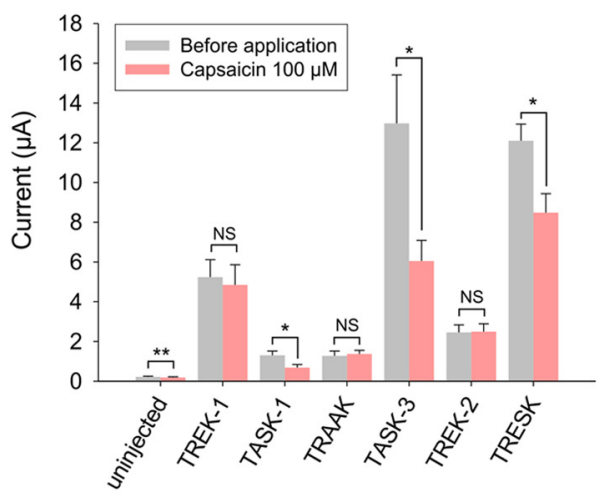

B
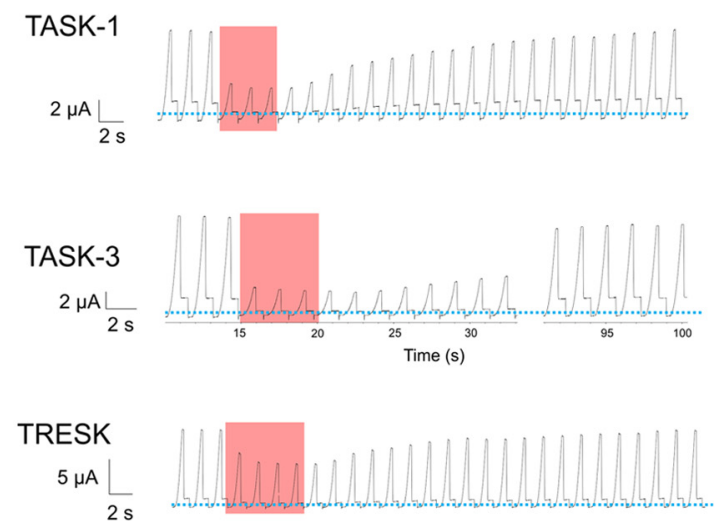

- Capsaicin $100 \mu \mathrm{M}$

E

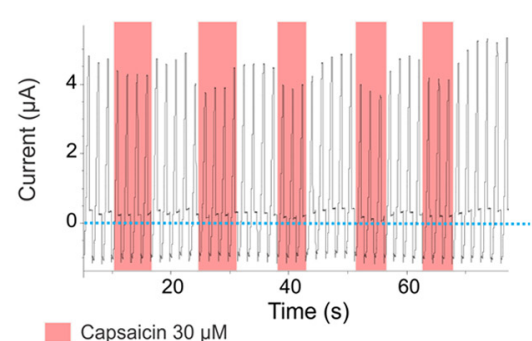

FIGURE 3 | (A) Left (from left to right) absolute currents presented by: uninjected Xenopus oocytes, oocytes injected with cRNA coding for hTREK-1, hTASK-1, hTRAAK, hTASK-3, hTREK-2 and hTRESK, before and after the application of $100 \mu \mathrm{M}$ capsaicin. Right: normalized currents showing the effect of $100 \mu \mathrm{M}$ capsaicin on Xenopus oocytes injected with cRNA coding for hTREK-1, hTASK-1, hTRAAK, hTASK-3, hTREK-2 and hTRESK. For each group, the current was normalized to the current registered prior to the application of $100 \mu \mathrm{M}$ capsaicin (dotted line). All data are expressed as the mean $+1-$ SEM. $N$ of $3-6$ oocytes. ${ }^{*} P<0.05$, ${ }^{*} P<0.01$. (B) Representative voltage clamp recordings of Xenopus laevis oocytes expressing TASK-1 (top), TASK-3 (middle) and TRESK (bottom), before, during and after the application of $100 \mu \mathrm{M}$ capsaicin, showing in every case a fully reversible inhibition. Dotted line represents zero current.

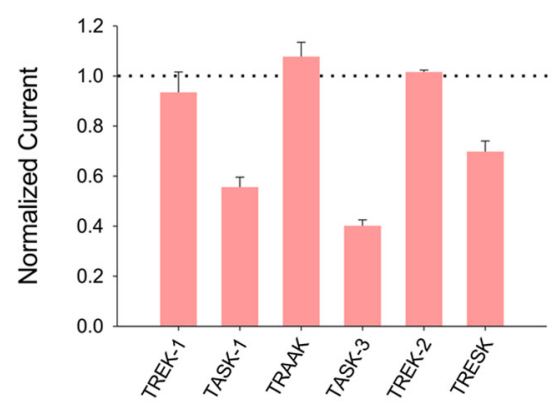

C
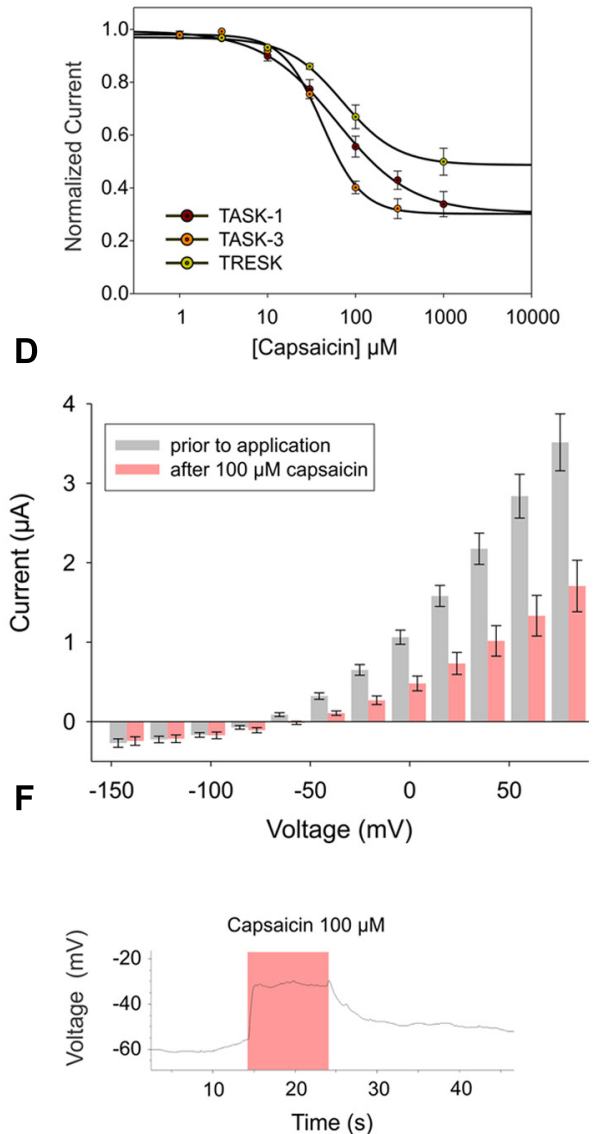

(C) Dose-response curves for capsaicin on TASK-1 (red circles), TASK-3 (orange circles) and TRESK (green circles). The $I_{50}$ values are listed in the text and in Table 1. $\mathrm{N}$ of 3-6 oocytes. For each measurement, the current was normalized to the current registered prior to the application of capsaicin. (D) hTASK-3 currents recorded before and after the application of $100 \mu \mathrm{M}$ capsaicin. The currents were elicited by voltage pulses from -140 to $+80 \mathrm{mV}$ in $20 \mathrm{mV}$ steps, $500 \mathrm{~ms}$ duration from a holding potential of $-80 \mathrm{mV}$. Values were obtained during the last $50 \mathrm{~ms}$ of the voltage pulses. $\mathrm{N}$ of 6 oocytes. (E) Representative voltage clamp recording of a Xenopus oocyte expressing TASK-3 repetitively exposed to $30 \mu \mathrm{M}$ capsaicin. (F) Current clamp recording of a Xenopus oocyte expressing TASK-3 exposed to $100 \mu \mathrm{M}$ capsaicin, which led to a membrane depolarization of approximately $30 \mathrm{mV}$. 
capsaicin; however, the magnitude of the background current presented by non-injected oocytes, both before and after application, was negligible in comparison to the current presented by injected oocytes (Figure 3A).

Capsaicin strongly and reversibly inhibited the high background current possessed by TASK-1, TASK-3 and TRESKinjected Xenopus laevis oocytes (Figure 3B). All three channels were inhibited by capsaicin, with $\mathrm{IC}_{50}$ values in approximately the same range: $41.8 \pm 3.6 \mu \mathrm{M}, 61.1 \pm 4.0 \mu \mathrm{M}$ and $70.2 \pm 5.3 \mu \mathrm{M}$ for TASK-3, TASK-1 and TRESK, respectively (Figure 3C and Table 1). Similarly to what we observed for piperine on TASK1, the inhibitory effect of capsaicin upon TASK-3 was more pronounced at potentials more positive than the potassium reversal potential (Figure 3D) and the channel behaved similarly when exposed to repetitive applications of capsaicin (Figure 3E). Additionally, this inhibition of potassium efflux markedly changed the membrane voltage towards more positive potentials as could be observed in the current-clamp mode (Figure 3F).

\section{6-Gingerol}

Continuing with our screening of alimentary pungent substances, we studied the effect of the ginger component 6-gingerol and found that it induced an inhibition that was fully reversible and dose-dependent on TASK-1, TASK-3, and TRESK, respectively (Figures 4A-C). Interestingly, $300 \mu \mathrm{M}$ of 6-gingerol significantly

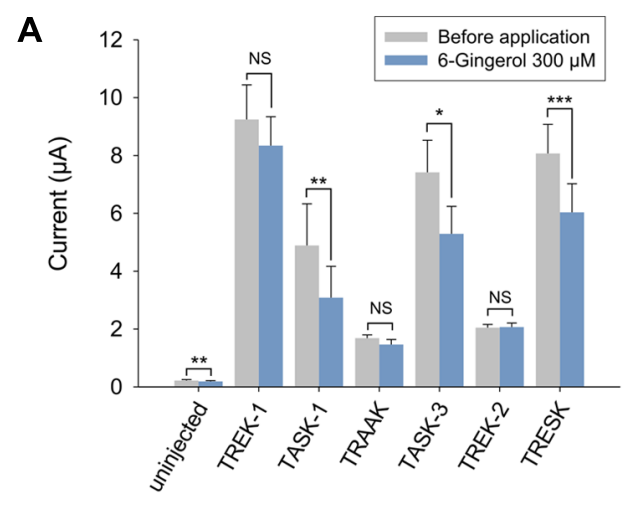

B

TASK-1

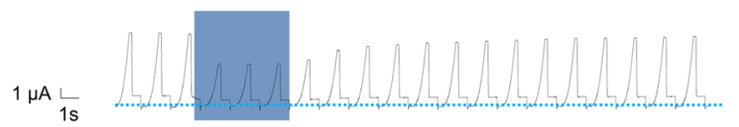

TASK-3

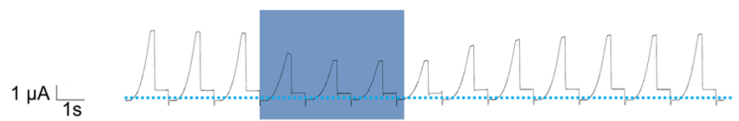

TRESK

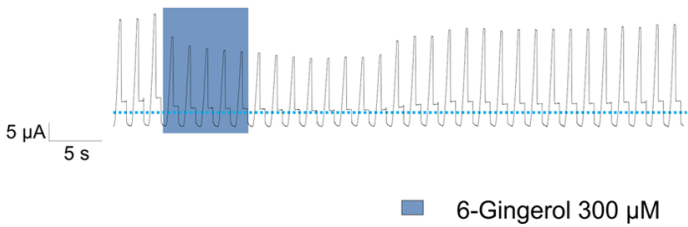

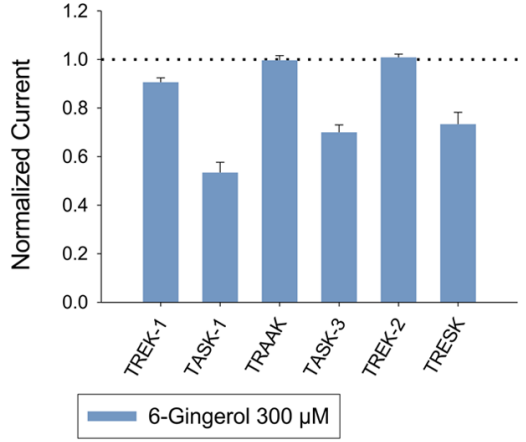

C

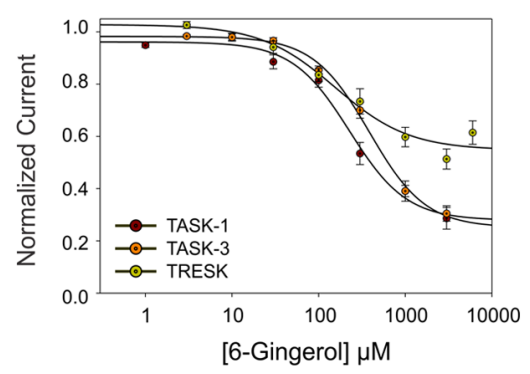

mean + /- SEM. N of 9-18 oocytes. ${ }^{*} P<0.05$, ${ }^{*} P<0.01$, ${ }^{*}{ }^{*} P<0.001$ (B) Representative voltage clamp recordings of Xenopus laevis oocytes expressing TASK-1 (top), TASK-3 (middle) and TRESK (bottom), before, during and after the application of $300 \mu \mathrm{M} 6$-gingerol, showing fully reversible inhibition in all cases. Dotted line represents zero current. (C) Dose-response curves for 6-gingerol on TASK-1 (red circles), TASK-3 (orange circles) and TRESK (green circles). N of 3-19 oocytes. For each measurement, the current was normalized to the current registered prior to the application of 6-gingerol. 
inhibited the endogenous background current presented by uninjected Xenopus oocytes as already observed for capsaicin. Again, the magnitude of the background current before and after the application of $300 \mu \mathrm{M}$ 6-gingerol, was negligible when compared to the background current presented by Xenopus oocytes expressing any of the evaluated $\mathrm{K}_{2 \mathrm{P}}$ channels (Figure 4A). All three channels were inhibited by 6-gingerol in a dose-dependent manner, with $\mathrm{IC}_{50}$ values of $155.0 \pm 70.9 \mu \mathrm{M}, 227.4 \pm 46.5 \mu \mathrm{M}$ and $384.6 \pm 55.6 \mu \mathrm{M}$ for TRESK, TASK-1, and TASK-3 respectively (Figure 4C and Table 1).

\section{Polygodial}

Lastly, we evaluated the effect of polygodial, the pungent key principle of tasmanian mountain pepper, on human $\mathrm{K}_{2 \mathrm{P}}$ channels. We observed that $1 \mathrm{mM}$ could significantly inhibit the basal activity of TASK-1, TASK-3 and, to a lesser degree, also TRESK (Figure 5A). Interestingly, the polygodial-induced inhibition of TASK-1 and TASK-3 was only partially reversible, even after a prolonged washout period (Figure 5B). TASK-1 and TASK-3 were both inhibited by polygodial in a dose-dependent manner, with $\mathrm{IC}_{50}$ values of $328 \pm 24.7 \mu \mathrm{M}$ and $484.5 \pm 18.2 \mu \mathrm{M}$ for TASK-3 and TASK-1, respectively (Figure 5C).

\section{The histidine residue at position 98 of TASK-3 is involved in the inhibitory effect induced by capsaicin, piperine and 6-gingerol}

After evaluating the effect of these pungent substances on $\mathrm{K}_{2 \mathrm{P}}$ channels, we investigated which amino acids could be involved in this interaction. To address this question, we constructed

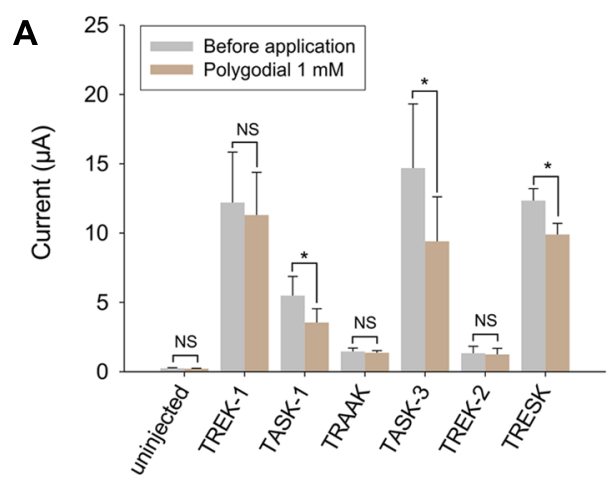

B
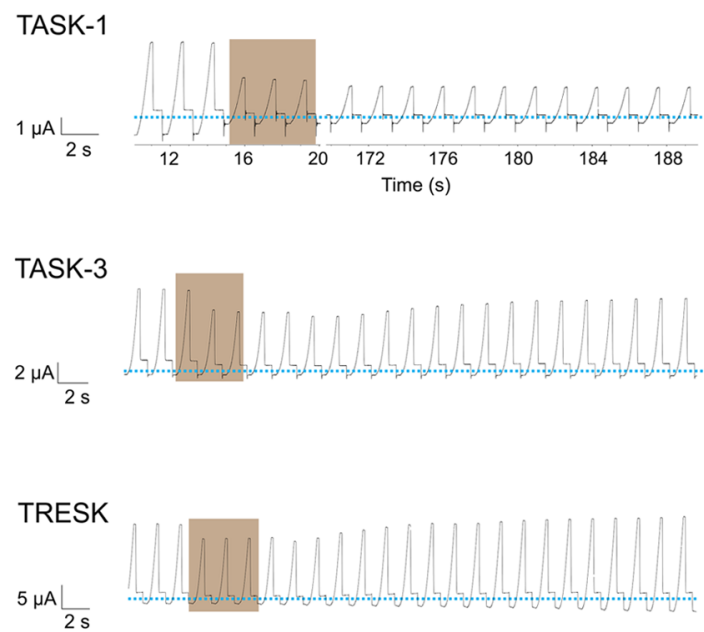

Polygodial $1 \mathrm{mM}$

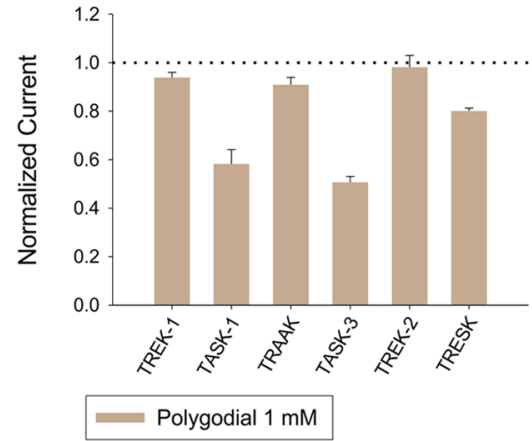

C

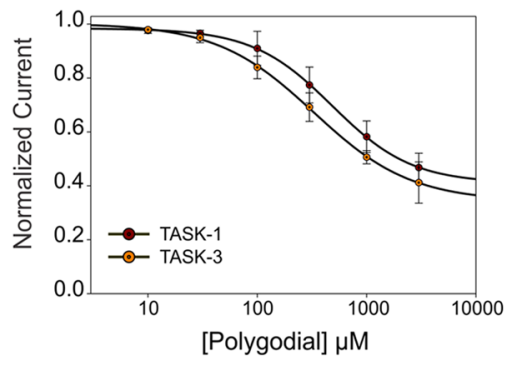
of Xenopus laevis oocytes expressing TASK-1 (top), TASK-3 (middle) and TRESK (bottom), before, during and after the application of $1 \mathrm{mM}$ polygodial, showing a partially reversible inhibition for TASK-1 and TASK-3. Dotted line represents zero current. (C) Dose-response curves for polygodial on TASK-1 (red circles) and TASK-3 (orange circles). The $\mathrm{IC}_{50}$ values are listed in the text and in Table 1. N of 3-5 oocytes. For each measurement, the current was normalized to the current registered prior to the application of polygodial. 
mutants for TASK-3, in which we targeted amino acids necessary for the interaction with well-described agonists or antagonists (Figure 6A).

First, the histidine at position 98, a key amino acid for the inhibition induced by protons (Rajan et al., 2000; Lopes et al., 2001), was replaced by glutamic acid. The H98E mutant showed significantly less inhibition by $100 \mu \mathrm{M}$ capsaicin, $300 \mu \mathrm{M}$ piperine and $1 \mathrm{mM}$ 6-gingerol; $1 \mathrm{mM}$ polygodial, however, had the same effect as on wild-type (Figure 6B). Piperine, capsaicin, and 6-gingerol, but not polygodial, induced a significantly decreased inhibitory effect at several other concentrations in the H98E mutant when compared with the wild type (Figures 7A-D). In view of this, we investigated whether simultaneous application of piperine and a slightly acidic $\mathrm{pH}$ could lead to an increased inhibition of TASK-3 WT. At pH 6.5, which markedly decreased the amount of current carried by TASK-3 but did not abolish it completely, no further inhibition could be observed after the application of $300 \mu \mathrm{M}$ piperine (Figure 6E). This was also the case for TASK-1, in which $300 \mu \mathrm{M}$ piperine at $\mathrm{pH} 6.5$ induced no further inhibition (not shown). We then constructed a chimera by replacing the VLRFLT $_{243-248}$ sequence of TASK-3, required for volatile anesthetics to induce their activating effect on this channel (Talley and Bayliss, 2002), by the WLRVLS $243-248$ sequence of human TREK-2. However, this chimera presented no significant difference compared to the wild type in response to any of the four substances (Figure 6C). Additionally, in order to investigate if

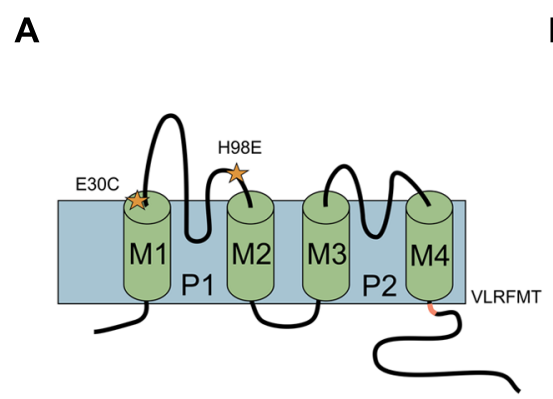

C

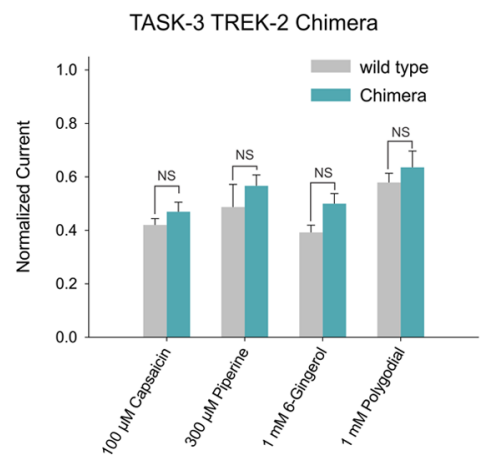

$\mathbf{E}$

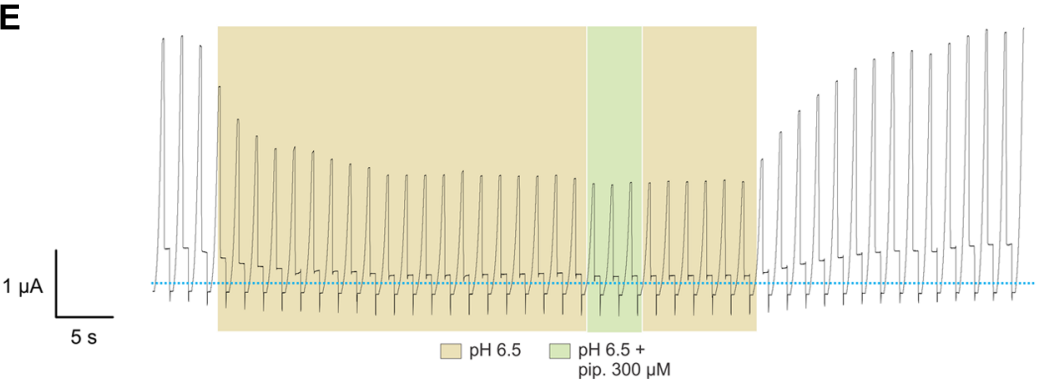

B

D
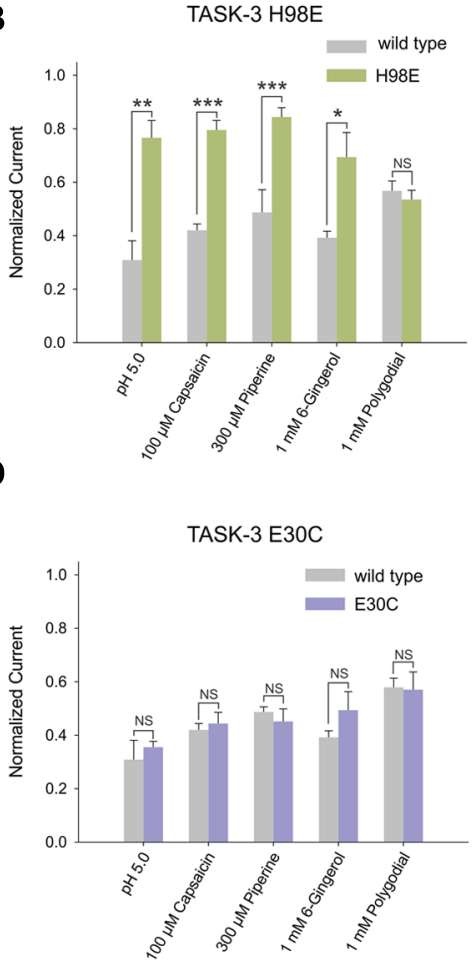

FIGURE 6 | (A) Human TASK-3 membrane topology diagram. Four transmembrane segments (M1 to $\mathrm{M} 4$ ) and two pore regions (P1 and P2) are shown. Mutated amino acids are indicated either with a star or as the replaced sequence. (B) Comparative effects of $\mathrm{pH}$ 5.0, $100 \mu \mathrm{M}$ capsaicin, $300 \mu \mathrm{M}$ piperine, $1 \mathrm{mM} 6$-gingerol and $1 \mathrm{mM}$ polygodial on wild-type human TASK-3 (gray bars) and the H98E mutant (green bars). $\mathrm{N}$ of 4-6 oocytes. ${ }^{*} P<0.05$, ${ }^{*} P<0.01,{ }^{* *} P<0.001$. (C) Comparative effects of the four pungent substances under evaluation in wild-type hTASK-3 (gray bars) vs. TREK-2 chimera (light blue bars). $\mathrm{N}$ of $4-5$ oocytes. (D) Comparative effects of $\mathrm{pH}$ 5.0, $100 \mu \mathrm{M}$ capsaicin, $300 \mu \mathrm{M}$ piperine, $1 \mathrm{mM}$ 6-gingerol and $1 \mathrm{mM}$ polygodial on wild-type human TASK-3 (gray bars) and the E30C mutant (purple bars). N of 4-6 oocytes. (E) Representative voltage clamp recordings of a Xenopus laevis oocyte expressing TASK-3 WT exposed to $\mathrm{a} \mathrm{pH}$ of 6.5 and then to $300 \mu \mathrm{M}$ piperine. 
A

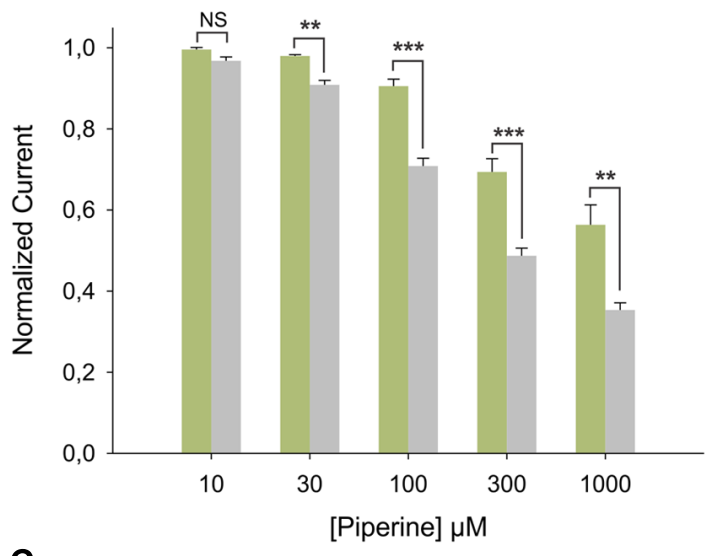

C

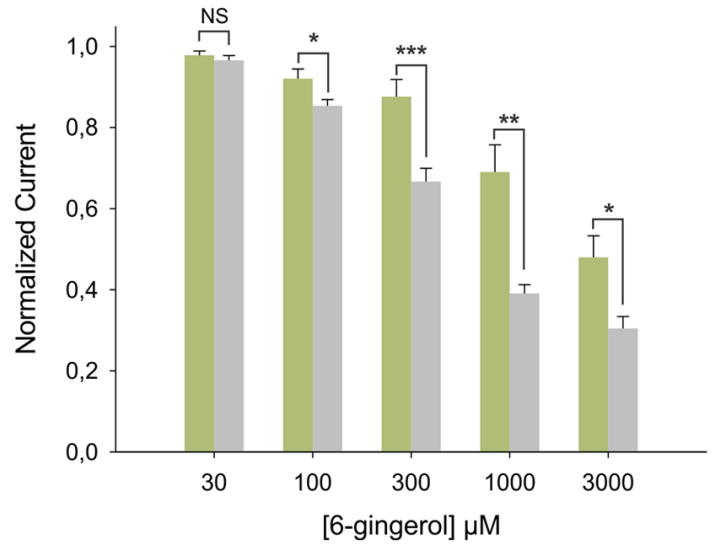

TASK-3 wild type

H98E

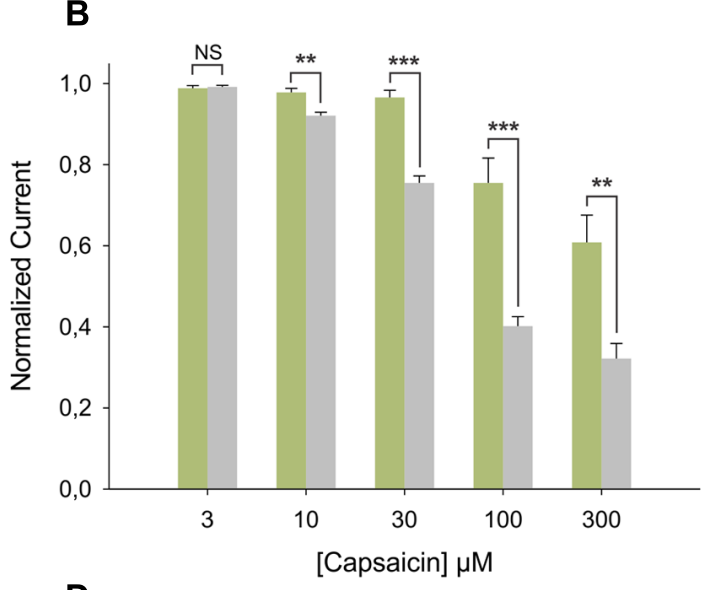

D

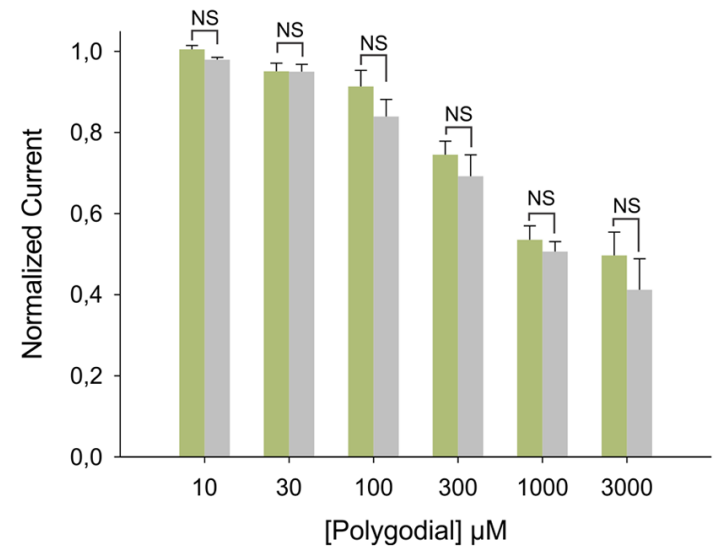

FIGURE 7 | Dose response relationships for the inhibition induced by pungent substances on TASK-3 WT (gray bars) vs. H98E (green bars) for (A) piperine, $\mathrm{N}$ of 5-12 oocytes; (B) capsaicin, $\mathrm{N}$ of 5-10 oocytes; (C) 6-gingerol, $\mathrm{N}$ of 3-19 oocytes and (D) polygodial $\mathrm{N}$ of 3-9 oocytes. ${ }^{*} P<0.05$, ${ }^{* *} P<0.01,{ }^{* *} P<0.001$.

these pungent substances bind preferentially to a closed state of the channel, as it is the case for $\mathrm{Zn}^{2+}$, we constructed an E30C mutant, which is reported to induce a closed state in TASK-3 (Veale et al., 2005; Mathie et al., 2010). This mutation, however, produced no significant change in the efficacy of the inhibition induced by any of the four substances at the tested concentrations (Figure 6D).

\section{DISCUSSION}

In the present work, we have expanded the pharmacological knowledge about $\mathrm{K}_{2 \mathrm{P}}$ channels. We have shown that the background $\mathrm{K}^{+}$current carried by the human $\mathrm{K}_{2 \mathrm{P}}$ channels TASK-1, TASK-3, and TRESK can be inhibited by piperine, the pungent principle of black pepper, whose mechanism of action has been thought to reside only on its effect on TRPV1. Interestingly this substance presents an $\mathrm{IC}_{50}$ on TASK-1 that is similar to its reported $\mathrm{EC}_{50}$ on TRPV1-37.9 $\pm 1.9 \mu \mathrm{M}$ when evaluated on transfected HEK293 cells (McNamara et al., 2005). The other 3 pungent substances investigated, namely capsaicin, 6-gingerol, and polygodial, also exerted an inhibitory effect, although at concentrations that were almost an order of magnitude higher than their $\mathrm{EC}_{50}$ values on TRP channels (Caterina et al., 1997; McNamara et al., 2005; Iwasaki et al., 2006).

Our results call for further studies in order to determine whether $\mathrm{K}_{2 \mathrm{P}}$ channels also play physiological roles in the perception of piperine and other pungent substances. Thus, to investigate the effect of pungent substances on trigeminal ganglia neurons incubated with TRP blockers would be a plausible next step. Moreover, experimental studies are needed to answer the question as to whether these three $\mathrm{K}_{2 \mathrm{P}}$ channels co-express with voltage gated sodium channels, such as $\mathrm{Na}_{\mathrm{v}} 1.8$, which would react to such a depolarizing event, or with TRP channels, whose activating effect would be facilitated by the simultaneous inhibition of $\mathrm{K}_{2 \mathrm{P}}$ channels. It is also worth noticing that the existence of additional players in the perception of piperine and capsaicin, is suggested by findings such as the stimulus-induced recovery (Green, 1998), and since $\mathrm{K}_{2 \mathrm{P}}$ channels do not change the magnitude of their response to any of the tested pungent substances after repeated 
applications, they deserve to be further studied in regard to this stimulus-induced recovery.

Our mutant studies showed that, when His98 is mutated to Glu, the TASK-3 responses to piperine, capsaicin and 6-gingerol are still present, but significantly reduced at most of the tested concentrations. Interestingly, this amino acid is thought to be the main proton sensor in TASK-1 and TASK-3 (Rajan et al., 2000; Lopes et al., 2001), and, when mutated, leads to a marked change in proton sensitivity (Rajan et al., 2000). For TASK-1 the postulated mechanism involves a water molecule behind the selectivity filter, which interacts with His 98 and other 3 amino acids in order to stabilize the backbone of the selectivity filter. According to this model, when His98 is protonated, it rotates up and consequently an electropositive barrier to $\mathrm{K}^{+}$at the outer mouth of the channel is created (Stansfeld et al., 2008). The large molecular size of capsaicin, piperine, and 6-gingerol makes it difficult to suggest that they might inhibit TASK-3 by means of the same mechanism. However, the fact that the same concentrations of the above mentioned substances induced a significantly greater inhibition in WT in comparison to the His 98 mutant, strongly suggest that this amino acid might play a role in the inhibition induced by these phytochemicals.

\section{ACKNOWLEDGMENT}

This work was funded by a grant (SFB 874) from the German Research Foundation (Deutsche Forschungsgemeinschaft) to Hanns Hatt (TP A3).

\section{AUTHOR CONTRIBUTIONS}

Conceived and designed the experiments: Hanns Hatt, Thomas Hofmann, Guenter Gisselmann, Leopoldo R. Beltrán, Corinna Dawid, Madeline Beltrán. Performed the experiments: Leopoldo R. Beltrán, Corinna Dawid, Madeline Beltrán, Katharina Degenhardt, Klaus Mathie. Analyzed the data: Leopoldo R. Beltrán, Madeline Beltrán. Wrote the paper: Leopoldo R. Beltrán, Corinna Dawid, Madeline Beltrán. Leopoldo R. Beltrán, Corinna Dawid, Madeline Beltrán contributed equally to the present work.

\section{REFERENCES}

André, E., Campi, B., Trevisani, M., Ferreira, J., Malheiros, Â., Yunes, R. A., et al. (2006). Pharmacological characterization of the plant sesquiterpenes polygodial and drimanial vanilloid receptor agonists. Biochem. Pharmacol. 71, 1248-1254. doi: 10.1016/j.bcp.2005.12.030

Bandell, M., Story, G. M., Hangs, S. W., Viswanath, V., Eid, S. R., Petrus, M. J., et al. (2004). Noxious cold ion channel TRPAl is activated by pungent compounds and bradykinin. Neuron 41, 849-857. doi: 10.1016/S0896-6273(04)00150-3

Barnes, C. S., and Loder, J. W. (1962). The structure of polygodial: a new sesquiterpene dialdehyde from polygonum hydropiper L. Austr. J. Chem. 15, 322-327. doi: 10.1071/CH9620322

Bautista, D. M., Sigal, Y. M., Milstein, A. D., Garrison, J. L., Zorn, J. A., Tsuruda, P. R., et al. (2008). Pungent agents from Szechuan peppers excite sensory neurons by inhibiting two-pore potassium channels. Nat. Neurosci. 11, 772-779. doi: 10.1038/nn.2143

Belitz, H. D., Grosch, W., and Schieberle, P. (2004). Food Chemistry, 3rd Edn . Berlin: Springer Verlag. doi: 10.1007/978-3-662-07279-0

Beltrán, L., Beltrán, M., Aguado, A., Gisselmann, G., and Hatt, H. (2013). 2-Aminoethoxydiphenyl borate activates the mechanically gated human KCNK channels KCNK 2 (TREK-1), KCNK 4 (TRAAK) and KCNK 10 (TREK-2). Front. Pharmacol. 4:63. doi: 10.3389/fphar.2013.00063
Calixto, J. B., Kassuya, C. A., André, E., and Ferreira, J. (2005). Contribution of natural products to the discovery of thr transient reporter potential (TRP) channels family and their functions. Pharmacol. Ther. 106, 179-208. doi: 10.1016/j.pharmthera.2004.11.008

Caterina, M. J., Schumacher, M. A., Tominaga, M., Rosen, T. A., Levine, J. D., and Julius, D. (1997). The capsaicin receptor: a heat-activated ion channel in the pain pathway. Nature 23, 816-824. doi: 10.1038/39807

Correa, E. A., Högestätt, E. D., Sterner, O., Echeverri, F., and Zygmunt, P. M. (2010). In vitro TRPV1 activity of piperine derived amides. Bioorg. Med. Chem. 18, 3299-3306. doi: 10.1016/j.bmc.2010.03.013

Dawid, C., Henze, A., Frank, O., Glabasnia, A., Rupp, M., Büning, K., et al. (2012). Structural and sensory characterization of key pungent and tingling compounds from black pepper (Piper nigrum L.). J. Agric. Food Chem. 60, 2884-2895. doi: 10.1021/jf300036a

Dedov, V. N., Tran, V. H., Duke, C. C., Connor, M., Christie, M. J., Mandadi, S., et al. (2002). Gingerols: a novel class of vanilloid receptor (VR1) agonists. Br. J. Pharmacol. 137, 793-798. doi: 10.1038/sj.bjp.0704925

Escalera, J., von Hehn, C. A., Bessac, B. F., Sivula, M., and Jordt, S. E. (2008). TRPA1 mediates the noxious effects of natural sesquiterpene deterrents. J. Biol. Chem. 283, 24136-24144. doi: 10.1074/jbc.M710280200

Enyedi, P., and Czirják, G. (2010). Molecular background of leak K+ currents: two-pore domain potassium channels. Physiol. Rev. 90, 559-605. doi: 10.1152/physrev.00029.2009

Green, B. G. (1996). Rapid recovery from capsaicin desensitization during recurrent stimulation. Pain 68, 245-253. doi: 10.1016/S0304-3959(96)03211-3

Green, B. G. (1998). Capsaicin desensitization and stimulus-induced recovery on facial compared to lingual skin. Physiol. Behav. 65, 517-523. doi: 10.1016/S00319384(98)00202-9

Goldstein, S., Bockenhauer, D., O’Kelly, I., and Zilberberg, N. (2001). Potassium leak channels and the KCNK family of two-P-domian subunits. Nat. Rev. Neurosci. 2, 175-184. doi: 10.1038/35058574

Iwasaki, Y., Morita, A., Iwasawa, T., Kobata, K., Sekiwa, Y., Morimitsu, Y., et al. (2006). A nonpungent component of steamed ginger - [10]-shogaol - increases adrenaline secretion via the activation of TRPV1. Nutr. Neurosci. 9, 169-178. doi: 10.1080/10284150600955164

Iwasaki, Y., Tanabe, M., Kayama, Y., Abe, M., Kashio, M., Koizumi, K., etal. (2009). Miogadial and miogatrial with alpha,beta-unsaturated 1,4dialdehyde moieties-novel and potent TRPAl agonists. Life Sci. 85, 60-69. doi: 10.1016/j.lfs.2009.04.017

Lopes, C. M., Zilberberg, N., and Goldstein, S. A. (2001). Block of Kcnk3 by protons. Evidence that 2-P-domain potassium channel subunits function as homodimers. J. Biol. Chem. 276, 24449-24452. doi: 10.1074/jbc.C100184200

Mathie, A., Al-Moubarak, E., and Veale, E. L. (2010). Gating of two pore domain potassium channels. J. Physiol. 588, 3149-3156. doi: 10.1113/jphysiol.2010.192344

McNamara, F. N., Randall, A., and Gunthorpe, M. J. (2005). Effects of piperine, the pungent component of black pepper, at the human vanilloid receptor (TRPV1). Br. J. Pharmacol. 144, 781-790. doi: 10.1038/sj.bjp.0706040

Morera, E., De Petrocellis, L., Morera, L., Moriello, A. S., Nalli, M., Di Marzo, V., et al. (2012). Synthesis and biological evaluation of [6]-gingerol analogues as transient receptor potential channel TRPV1 and TRPA1 modulators. Bioorg. Med. Chem. Lett. 22, 1674-1677. doi: 10.1016/j.bmcl.2011.12.113

Noël, J., Zimmermann, K., Busserolles, J., Deval, E., Alloui, A., Diochot, S., et al. (2009). The mechano-activated K+ channels TRAAK and TREK-1 control both warm and cold perception. EMBO J. 28, 1308-1318. doi: 10.1038/emboj.2009.57

Okumura, Y., Narukawa, M., Iwasaki, Y., Ishikawa, A., Matsuda, H., Yoshikawa, M., et al. (2010). Activation of TRPV1 and TRPA1 by black pepper components. Biosci. Biotechnol. Biochem. 5, 1068-1072. doi: 10.1271/bbb.90964

Rajan, S., Wischmeyer, E., Xin Liu, G., Preisig-Müller, R., Daut, J., Karschin, A., et al. (2000). TASK-3, a novel tandem pore domain acid-sensitive K+ channel. An extracellular histiding as $\mathrm{pH}$ sensor. J. Biol. Chem. 275, 16650-16657. doi: 10.1074/jbc.M000030200

Stansfeld, P. J., Grottesi, A., Sands, Z. A., Sansom, M. S., Gedeck, P., Gosling, M., et al. (2008). Insight into the mechanism of inactivation and $\mathrm{pH}$ sensitivity in potassium channels from molecular dynamics simulations. Biochemistry 47, 7414-7422. doi: 10.1021/bi800475j

Staudacher, K., Baldea, I., Kisselbach, J., Staudacher, I., Rahm, A. K., Schweizer, P. A., et al. (2011). Alternative splicing determines mRNA translation initiation 
and function of human K(2P)10.1 K+ channels. J. Physiol. 589, 3709-3720. doi: 10.1113/jphysiol.2011.210666.

Talley, E. M., and Bayliss, D. A. (2002). Modulation of TASK-1 (Kcnk3) and TASK-3 (Kcnk9) potassium channels: volatile anesthetics and neurotransmitters share a molecular site of action. J. Biol. Chem. 277, 17733-17742. doi: 10.1074/jbc.M200502200

Veale, E. L., Clarke, C. E., and Mathie, A. (2005). A key glutamate residue (E30), conserved in many $\mathrm{K}$ channels, regulates the gating of TASK3 two pore domain potassium channels. J. Physiol. 567P, PC196(abstract).

Vogt-Eisele, A. K., Weber, K., Sherkheli, M. A., Vielhaber, G., Panten, J., Gisselmann, G., et al. (2007). Monoterpenoid agonists of TRPV3. Br. J. Pharmacol. 151, 530540. doi: $10.1038 /$ sj.bjp. 0707245

Witte, D. G., Cassar, S. C., Masters, J. N., Esbenshade, T., and Hancock, A. A. (2002), Use of a fluorescent imaging plate reader-based calcium assay pharmacological differences between the human and rat vaniloid receptor. J. Biomol. Scree. 7, 466-475.

Yamamoto, Y., Hatakeyama, T., and Taniguchi, K. (2009). Immunohistochemical colocalization of TREK-1, TREK-2 and TRAAK with TRP channels in the trigeminal ganglion cells. Neurosci. Lett. 454, 129-133.
Conflict of Interest Statement: The authors declare that the research was conducted in the absence of any commercial or financial relationships that could be construed as a potential conflict of interest.

Received: 15 August 2013; accepted: 23 October 2013; published online: 18 November 2013.

Citation: Beltrán LR, Dawid C, Beltrán M, Gisselmann G, Degenhardt K, Mathie $K$, Hofmann $T$ and Hatt $H$ (2013) The pungent substances piperine, capsaicin, 6gingerol and polygodial inhibit the human two-pore domain potassium channels TASK-

1, TASK-3 and TRESK. Front. Pharmacol. 4:141. doi: 10.3389/fphar.2013.00141

This article was submitted to Neuropharmacology, a section of the journal Frontiers in Pharmacology.

Copyright (e) 2013 Beltrán, Dawid, Beltrán, Gisselmann, Degenhardt, Mathie, Hofmann and Hatt. This is an open-access article distributed under the terms of the Creative Commons Attribution License (CC BY). The use, distribution or reproduction in other forums is permitted, provided the original author(s) or licensor are credited and that the original publication in this journal is cited, in accordance with accepted academic practice. No use, distribution or reproduction is permitted which does not comply with these terms. 